\title{
Corela
}

Cognition, représentation, langage

HS-13 | 2013

Statut et utilisation des corpus en linguistique

\section{L'acquisition du Present Perfect chez un enfant anglophone : méthodologie, codage et premiers résultats}

Laurent David

\section{OpenEdition}

Journals

Édition électronique

URL : http://journals.openedition.org/corela/3050

DOI : $10.4000 /$ corela.3050

ISSN : 1638-573X

Éditeur

Cercle linguistique du Centre et de l'Ouest - CerLICO

Référence électronique

Laurent David, "L'acquisition du Present Perfect chez un enfant anglophone : méthodologie, codage et premiers résultats », Corela [En ligne], HS-13 | 2013, mis en ligne le 10 décembre 2013, consulté le 20 avril 2019. URL : http://journals.openedition.org/corela/3050 ; DOI : 10.4000/corela.3050

Ce document a été généré automatiquement le 20 avril 2019.

\section{(c) (i) (2)(2)}

Corela - cognition, représentation, langage est mis à disposition selon les termes de la licence Creative Commons Attribution - Pas d'Utilisation Commerciale - Partage dans les Mêmes Conditions 4.0 International. 


\title{
L'acquisition du Present Perfect chez un enfant anglophone: méthodologie, codage et premiers résultats
}

\author{
Laurent David
}

\section{Introduction}

1 Dans cet article sont exposés la démarche et les choix méthodologiques effectués pour une étude de l'acquisition du Present Perfect (désormais PP), composé de l'auxiliaire HAVE et du participe passé du verbe lexical, par des enfants anglophones.

2 À terme, cette étude a pour but de comprendre comment s'opère la construction des valeurs du PP dans l'esprit d'un enfant en cours d'acquisition du langage, quelle est la nature de cette construction (question de l'inné et de l'acquis, influence de l'input et du contexte extralinguistique, acquisition par constructions liées à des contextes précis), comment elle s'inscrit dans l'esprit de l'enfant et comment elle se diversifie et se rattache à des conceptualisations de plus en plus complexes.

3 Ce travail s'appuie sur un corpus oral dont nous allons, dans un premier temps, expliquer le choix en regard des approches théoriques sélectionnées. Dans un deuxième temps, nous nous intéresserons au protocole mis en place ainsi qu'à l'élaboration du codage. Suite aux travaux récents de l'équipe CoLaJe (http://colaje.risc.cnrs.fr, Morgenstern, 2009), nous estimons essentiel de faire des analyses quantitatives et qualitatives multimodales. Nous présenterons dans un dernier temps les premiers résultats obtenus et les questions qu'ils suscitent. 


\section{Approches théoriques sélectionnées}

4 La littérature concernant l'aspect et plus particulièrement l'aspect grammatical abonde. Avoir recours à une forme grammaticale spécifique telle que le PP pour décrire un événement suscite de nombreux questionnement. Depuis les considérations d'Aristote dans la Métaphysique sur les différents types de procès jusqu'aux avancées récentes de la linguistique ou de la psychologie cognitive, ce domaine est un terrain de recherche fertile où différents cadres théoriques se côtoient et se nourrissent.

5 Pour cette raison, il est impossible de retenir toutes les approches théoriques existantes pour cette étude. Nous avons retenu cinq approches :

\subsection{La théorie de la pertinence (Benveniste, 1966 ; McCoard, 1978)}

6 McCoard considère que le PP exprime le passé d'un événement ayant un lien pertinent avec le $T_{0}$ de l'énonciateur. Cette théorie découle clairement de celle de Benveniste (1966) pour qui le «parfait [français] établit un lien vivant entre l'événement passé et le présent où son évocation trouve place » (p.244). La théorie de la pertinence donne une définition assez large et ce lien entre le passé et $\mathrm{T}_{0}$ mérite donc d'être plus détaillé.

\subsection{La catégorisation de Comrie (1976) en quatre valeurs sémantiques}

résultat: I have lost my keys (and I cannot open the door).

expérience: I have been in London four times.

situation persistante: I have known her since I was a child.

passé récent: I have just met him.

7 L'existence de ces quatre valeurs «stables », bien qu'elles soient nommées différemment chez d'autres linguistes (Leech, 1971; McCawley, 1971) permet un étiquetage simple des énoncés qui permet déjà un premier regroupement.

\subsection{Une théorie basée sur le discours (Nishiyama \& Koening, 2005)}

Il semble intéressant d'envisager le PP comme un choix stratégique de l'énonciateur puisque notre étude s'appuie sur un corpus conversationnel. Nishiyama \& Koening (2005) se fondent sur deux corpus anglais et japonais regroupant plusieurs genres (presse, discussion, conversation, narration) et montrent le rôle joué par le PP (en opposition au prétérit simple) dans la mise en place des relations discursives et de la cohérence textuelle. Les auteurs reviennent sur le fait que l'idée du «lien pertinent » ne met en valeur que la dimension temporelle ou causale du PP, le PP est soit un lien avec le passé récent, soit l'expression d'un rapport de cause/conséquence. Ils indiquent également que sa définition varie selon les linguistes ce qui rend sa nature encore plus ambiguë. Ils élaborent alors une autre théorie qui met en jeu la sémantique et la pragmatique, ils s'écartent du temporel et du causal. Selon eux, le PP impose la présence d'une variable sémantique $\mathrm{X}$ dont la valeur est retrouvée grâce à des inférences pragmatiques. Ils partent donc du principe que le discours joue un rôle crucial dans l'interprétation du PP : 
c'est finalement plus ce qu'il y a autour du PP qui va déterminer son interprétation que le sémantisme de la forme en elle-même.

Ils retiennent quatre types de PP :

Type 1. «entailed resultative » (résultatif inféré) : l'événement décrit a été validé dans le passé récent et a des conséquences sur $\mathrm{T}_{0}$;

Type 2. "evidential use » (usage évidentiel): avec des verbes performatifs ou épistémiques (say, promise, see) qui décrivent un événement qui sera validé dans le futur grâce aux conditions de vérité de l'acte de langage ;

Type 3. « topic negociation » (négociation du thème) : au début d'une conversation, l'énonciateur veut savoir si le co-énonciateur connaît le sujet de la conversation pour pouvoir en parler, l'énonciateur questionne l'expérience du co-énonciateur en utilisant du PP ;

Type 4. « commonsense entailment » (inférence cotextuelle) : l'énonciateur utilise le PP pour indiquer qu'un événement trouvé dans le co-texte droit ou gauche garantit la vérité de son énoncé.

Dans ces quatre types, la variable sémantique $\mathrm{X}$ est de nature différente :

Type 1. C'est la situation d'énonciation $\mathrm{T}_{0}$ (lien persistent) ;

Type 2. Ce sont les conditions de vérité de l'acte de langage qui garantissent la validation de l'événement décrit (on s'appuie sur la sincérité de l'énonciateur que l'on considère comme une source fiable) ;

Type 3. C'est le sujet de la conversation donné par l'énonciateur, ce dont il veut parler ;

Type 4. C'est un énoncé du co-texte gauche ou droit.

10 Les linguistes considèrent que le choix d'un PP pour décrire un événement qui a été validé ou a commencé dans le passé s'explique par la volonté de l'énonciateur d'expliquer et transmettre la cohérence discursive au co-énonciateur; le PP a donc un rôle dans cette cohérence, avec trois fonctions pragmatiques selon le type de PP :

1. ajouter de nouvelles relations discursives dans le discours, entre le passé et le présent (type 1), entre ce qui est dit et ce qui est/sera vrai (type 2);

2. négocier un thème (type 3 ) ;

3. aider à la cohérence du discours en lui-même, le PP signale qu'il faut retrouver

un autre énoncé dans le co-texte qui légitime son existence (type 4).

\subsection{Amorçage sémantique et représentation mentale des événements}

11 Le terme d'amorçage est emprunté à la psychologie cognitive. C'est une mémoire implicite due à l'exposition du sujet à un stimulus (l'amorce) qui va influencer un autre stimulus (la cible). L'effet d'amorçage est reconnu lorsque le temps de réaction à la cible est plus court chez le sujet ayant été exposé à l'amorce. Lorsque ce terme est appliqué à la sémantique, on part du principe qu'un sujet à qui l'on exposerait, par exemple, le mot bread puis butter aura un temps de réaction plus rapide qu'un autre sujet à qui l'on exposerait le mot bread puis doctor, ces deux termes n'appartenant pas au même réseau sémantique. En sémantique, lorsqu'un concept est activé, cela a pour résultat l'activation de tous les réseaux et les concepts reliés à ce dernier (Collins \& Loftus, 1975). La mémoire sémantique est organisée de façon à permettre un accès rapide et facile à la représentation des événements (Lancaster \& Barsalou, 1997; Vu, Kellas, Petersen, \& Metcalf, 2003). Ce processus met à disposition de larges données sémantiques reliées entre elles qui seraient utilisées lors de l'acquisition du langage de manière plus significative que le prétendent les approches basées uniquement sur le lexique. On 
considère qu'une représentation ou simulation mentale d'un événement contient des caractéristiques telles que le type d'événement, l'agent, la cause, le type d'action, le lieu, le but et le contexte temporel. Ces modèles peuvent évoluer lorsqu'ils sont confrontés à de nouvelles informations dans le discours, ce qui est intéressant pour notre étude conversationnelle. Madden \& Zwaan (2003) mettent l'accent sur l'importance des formes linguistiques qui jouent un rôle dans les représentations mentales car elles peuvent les changer. Les marqueurs aspectuels (le PP par exemple) influencent ainsi les représentations mentales et les caractéristiques liées qui vont être retenues. Selon eux, le PP restreint la représentation de l'événement à un état de complétude.

\subsection{Acquisition de l'aspect}

12 Le développement morpho-syntaxique est examiné dans des études générales sur le développement du domaine verbal (Brown, 1973 ; Weist, 1991; Wagner, 2001 ; Parisse \& Morgenstern, 2012) et dans des travaux sur la morphologie de l'inflexion -ED (Bronckart \& Sinclair 1973; Bloom, Lifter, Hafitz 1980; Antinucci \& Miller 1976). Chez certains auteurs, l'hypothèse de «l'aspect avant le temps » est mise en avant, c'est-à-dire qu'ils considèrent que l'inflexion -ED n'est pas utilisée par l'enfant pour coder un décrochage temporel mais pour marquer la complétude d'un événement. Shirai \& Andersen (1995) soulignent l'importance de l'aspect lexical en montrant que chez l'enfant, la marque morphologique du passé est principalement utilisée avec des verbes téliques (contrairement à la marque du progressif qui tend à être utilisé avec des verbes d'activité) et que cela s'explique par un effet de miroir par rapport à l'adulte. Les interactions entre l'aspect grammatical et l'aspect lexical en acquisition sont d'ailleurs examinés dans Bardovi-Harlig (2000) et Li \& Shirai (2000) à l'instar de la théorie développée par Smith (1991) où elle examine les relations et les interactions entre le Point de vue (Viewpoint), c'est-à-dire le perfectif ou l'imperfectif et le Type de situation (Situation Type) qui peut être un état ou un événement. Suite à l'hypothèse de "l'aspect avant le temps ", il existe un débat dans la littérature pour savoir si l'enfant utilise l'aspect grammatical uniquement pour marquer l'aspect ou pour marquer le temps également. L'étude longitudinale que nous allons mener permettra peut-être d'éclaircir ce point. En effet, Weist (1991, Weist et al. 1991, 1997) et Wagner $(1997,2001,2002)$ fournissent des éléments montrant qu'entre l'âge de 1 an et 5 ans, une progression existe dans l'usage et dans la compréhension de l'aspect verbal; elle peut même s'étendre au delà de 5 ans. Weist (2002) comme Tomasello (2003) considèrent que l'enfant ne possède pas l'intégralité de ses compétences langagières avant l'âge de 8 ans. Cette idée de développement est très importante dans cette étude car elle encourage à choisir un corpus longitudinal très dense qui s'étend au moins jusqu'à l'âge de 5 ans pour examiner au plus près tous les développements touchant le PP, qu'ils soient morphologiques, syntaxiques, sémantiques ou pragmatiques.

\section{Corpus et méthodologie}

\subsection{Choix du corpus}

13 L'étude de la littérature permet de faire plusieurs remarques qui influent sur le choix du corpus. Selon les études psycholinguistiques il semble plus intéressant d'étudier des 
enfants âgés de 2 ans à au moins 5 ans. Concernant le type de corpus, un corpus longitudinal très dense est recommandé pour pouvoir avoir un maximum d'occurrences $\mathrm{du}$ PP dans le but d'apprécier les développements morphologiques, syntaxiques et pragmatiques. Concernant le dialecte, il est mentionné dans un article de Slobin (1994) qu'en anglais américain, le PP est devenu «trop marginal pour une analyse adéquate de ses fonctions précoces»(p.120). Slobin mentionne également les travaux de Cromer (1968) et de Nussbaum \& Narremore (1975) qui indiquent que l'utilisation du PP par les enfants américains n'est pas significative avant l'âge de 5 ans. Cette affirmation peut être cependant nuancée par les travaux de Hundt \& Smith (2009) sur l'évolution du PP en anglais britannique et américain qui ne concluent pas à un déclin du PP en anglais américain mais plutôt à une relative stabilité dans les variations régionales (p.57). L'anglais britannique semble néanmoins offrir plus de possibilités pour cette étude.

Recherchant une certaine spontanéité dans l'usage de la langue, il est préférable de se tourner vers un corpus oral avec les pistes audio disponibles. Pour étudier l'acquisition, nous avons choisi la base de données du système Child Language Data Exchange System (http://childes.psy.cmu.edu, MacWhinney, B., 2000. The CHILDES Project: Tools for Analyzing Talk. Mahwah, NJ: Lawrence Erlbaum Associates). Elle comporte de nombreux corpus transcrits avec parfois les fichiers audio et vidéo qui leur correspondent. Le CHILDES propose aussi un logiciel de transcription adapté à l'anglais, le CHAT (Codes for the Human Analysis of Transcripts).

Deux enfants issus du projet CHILDES ont été choisis :

- Lara (Rowland, C. F. \& Fletcher, S. L. ; 2006), née et élevée à Nottinghamshire (Nord de l'Angleterre). Ce corpus présente 120 heures de conversation obtenues entre 1 an 9 mois et 3 ans 3 mois.

- Thomas (Lieven, E., Salomo, D. \& Tomasello, M. ; 2009), né en Angleterre. Ce corpus présente plus de 120 heures de conversation et a été recueilli entre 2 ans et 5 ans inclus.

Les pistes audio sont disponibles pour les deux enfants ainsi que les transcriptions détaillées en format CLAN.

Dans le cadre de cet article, seul Thomas sera étudié.

\subsection{Construction du protocole}

17 CLAN présente plusieurs avantages (Morgenstern, A., Parisse, C., 2007.) :

- la transcription est en phonétique, ce qui permet d'avoir une transcription très fidèle aux paroles de l'enfant;

- le non-verbal est explicité, c'est-à-dire que la transcription est annotée lorsque les locuteurs font un mouvement quel qu'il soit. Cela est utilisé pour palier l'absence de vidéo ;

- CLAN dispose d'un comptage d'occurrences; on peut rechercher facilement les formes et savoir quel pourcentage elles représentent par rapport aux énoncés totaux ;

- il y a une ligne réservée à l'analyse morpho-syntaxique (MOR) : les éléments de l'énoncé sont étiquetés en parties du discours. Cela est très utile pour notre étude car c'est la combinaison auxiliaire HAVE + participe passé qui est recherchée. C'est à partir de cette ligne que les occurrences du PP sont trouvées ;

- l'alignement simple avec l'audio est assuré grâce à un système de balises temporelles ; on peut à tout moment avoir accès à l'audio qui correspond à l'énoncé à étudier. 
CLAN dispose de nombreux programmes pour l'analyse des données brutes. Le programme KWAL est utilisé sur la ligne MOR pour trouver les occurrences de PP chez l'enfant, les co-textes gauche et droit (deux lignes à chaque fois) sont également donnés. La présence de l'audio fait partie intégrante du protocole puisqu'elle permet de vérifier la bonne distribution des tours de parole; on constate que l'alignement audio est parfois incorrect. L'audio peut aussi permettre d'analyser le développement phonologique de l'enfant même si cela ne s'intègre pas dans notre étude pour le moment. Cependant, l'étude de la prosodie (ligne mélodique) et des schémas accentuels sont plus abordables et peuvent révéler des indices quant à la maîtrise de la combinaison auxiliaire HAVE et verbe lexical.

Le codage des occurrences sous EXCEL se fait en deux temps. Dans un premier tableau, les formes sont isolées, elles sont numérotées avec l'âge, le numéro de ligne dans CLAN, les co-textes gauche et droit sont également copiés. Ce tableau n'a pas de visée analytique, il contient des données brutes mais il permet un repérage clair des formes relevées pour pouvoir y revenir rapidement et efficacement. Le deuxième tableau constitue une classification des formes, les données obtenues sont des méta-données :

- la morphologie : la forme correcte/incorrecte est codée ;

- la syntaxe : la syntaxe correcte/incorrecte est codée et tous les éléments qui font défaut sont notés (sujet non-exprimé, auxiliaire erroné, argument manquant) ;

- les formes de reprise : les formes qui sont initiées par l'enfant, dont l'enfant est à l'origine et les formes qu'il reprend (Yes I have ; No I haven't) sont distinguées ;

- l'expression de la possession (HAVE GOT) est incluse dans l'étude tout en les distinguant des autres formes de PP. Ce point est sujet à débat mais on parle souvent du PP en termes de résultat et d'expérience. Si l'on considère qu'exprimer ce que l'on possède à un moment donné revient à parler de son expérience et des résultats qu'elle provoque à un moment donné (l'énonciateur partage son expérience en disant ce qu'il possède), alors on peut considérer la forme HAVE GOT comme une instance de PP même si c'est une forme figée qui est utilisée exclusivement pour l'expression de la possession, qu'elle soit littérale ou métaphorique ;

- la valeur sémantique : elle est codée selon la catégorisation de Comrie (voir supra 1.2) ;

- la fonction pragmatique : elle est codée selon les quatre types de Nishiyama \& Koening (voir supra 1.3).

\section{Analyse d'une vignette}

Dans cette vignette, la mère de Thomas essaie de faire fonctionner un magnétophone. Thomas, âgé de 3 ans et 11 mois, assiste et participe à la scène.

MOT: It is definitely plugged in. Yes. So it should be working, shouldn't it?

CHI : I know what you haven't done. (1)

MOT: What?

CHI : You haven't speaked through it. (2)

MOT: I haven't speaked through it?

CHI : That's why it's not working. (3)

MOT: It's not speaked it's spoken.

$\mathrm{CHI}$ : It is not being spoken through. (4)

MOT: No you don't speak into anything. It is not like your tape recorder upstairs.

21 Thomas produit ici quatre énoncés complexes indiqués par les chiffres entre parenthèses, les énoncés en gras contiennent du PP. Nous pouvons déjà remarquer que la morpho- 
syntaxe est élaborée et quasi-correcte à l'exception de la régularisation du participe passé "spoken" en "speaked», phénomène fréquent à cet âge (Maslen et al., 2004). Le participe passé est d'ailleurs corrigé (" being spoken ») grâce à l'intervention explicite de la mère («it's not speaked it's spoken »). Pour analyser correctement ces énoncés, nous devons prendre en considération toute la scène pour pouvoir retrouver les liens discursifs qui expliquent le choix de ce PP.

Énoncé 1 : Il s'agit d'une structure enchâssée, le verbe cognitif « know » est le verbe de la principale ce qui montre que Thomas se base sur sa connaissance, son expérience pour affirmer quelque chose (I know something). Thomas se sert donc de son passé expérientiel pour faire un lien avec une situation présente (la situation d'énonciation), l'événement décrit (" what you haven't done ») est validé à $\mathrm{T}_{0}$, c'est le type 1 (résultat inféré) de la classification de Nishiyama \& Koening.

Cela souligne aussi l'importance de la mémoire événementielle: Thomas se sert d'un événement déjà vécu pour tenter d'en comprendre un autre de nature similaire.

Énoncé 2 : cet énoncé vient en réponse à l'interrogation de la mère («what») qui lui demande d'expliciter son propos. Le participe passé «done » utilisé par Thomas dans l'énoncé 1 fait référence à une activité mais sans pour autant en préciser la nature. L'énoncé 2 répond donc à ce manque de précision et donne une justification: si le magnétophone ne marche pas, c'est parce que sa mère ne lui a pas parlé. Le choix du PP a donc pour but d'aider à la cohérence du discours en lui-même, le co-texte gauche (« what you haven't done ») amorce ce processus qui se termine par l'énoncé 3 où Thomas exprime clairement que si le magnétophone ne marche pas c'est parce que («that's why ») sa mère ne lui a pas parlé.

On peut schématiser le parcours cognitif de Thomas comme ceci :

- le magnétophone ne marche pas; or Thomas a déjà vécu cette situation (si ce n'est qu'il confond le magnétophone avec l'enregistreur) ;

- il sait qu'il faut parler dans l'enregistreur (ici magnétophone) pour qu'il fonctionne or sa mère ne l'a pas fait ;

- il conclut qu'à $\mathrm{T}_{0}$, il y a quelque chose que sa mère n'a pas fait (« what you haven’t done »);

- il le lui signale (énoncé 1 ) et il explique son propos en donnant la raison pour laquelle selon lui le magnétophone ne marche pas (énoncé 2);

- il montre qu'il en est convaincu et qu'il a trouvé la bonne explication au problème (énoncé 3);

- l'énoncé 4 constitue une surenchère où Thomas se focalise sur le découpage interne de la situation d'énonciation avec une forme de présent progressif (« it is not being ») dans le but d'affirmer plus fermement et précisément son opinion. On sait d'ailleurs que le présent progressif constitue souvent un commentaire sur la situation d'énonciation.

L'analyse de cette vignette avait pour but de montrer comment les approches théoriques sont utilisées pour analyser les énoncés du corpus, nous pouvons maintenant aller vers leur regroupement pour en tirer des conclusions plus générales. 


\section{Premiers résultats (Thomas entre 2 et 4 ans)}

\subsection{Résultats quantitatifs}

Entre l'âge de 2 et 4 ans, on trouve 130 occurrences du PP. Nous avons organisé les résultats quantitatifs selon le codage (voir supra 2.2) : les formes en HAVE GOT sont séparées des formes HAVE +EN puis dans chacune de ces formes, nous avons distingué les formes de reprise et les formes initiées par l'enfant (initiation).

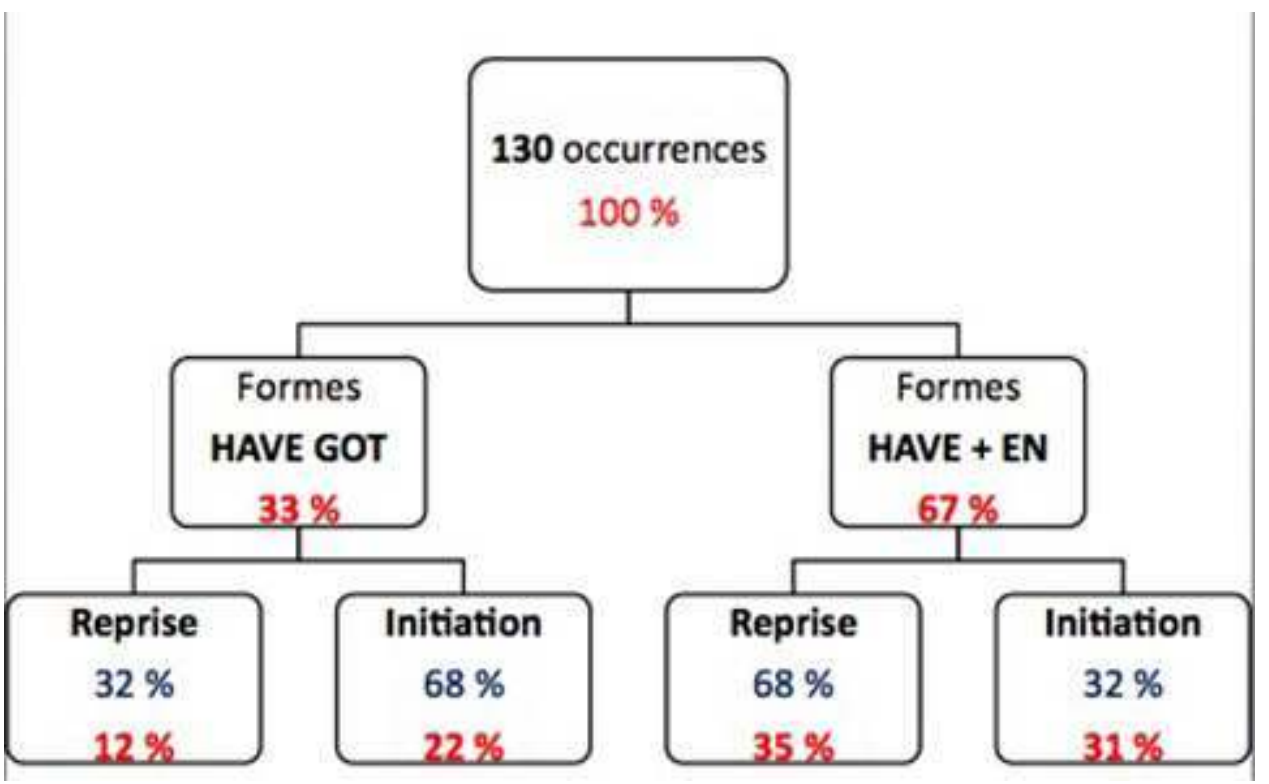

$\mathrm{Au}$ sein du nombre total d'occurrences, les formes en HAVE + EN sont majoritaires puisqu'elle représentent $67 \%$ du total. Cependant à l'intérieur de ces formes, seulement $32 \%$ sont initiées par l'enfant, $68 \%$ des formes sont donc des formes de reprise, ce qui souligne que Thomas ne dispose pas encore d'une syntaxe et d'une grammaire suffisante pour construire la majorité de ses énoncés : il réfléchit encore beaucoup par mimétisme et répétition, ce qui correspond entièrement aux problématiques de son âge dans l'acquisition du langage. En revanche, parmi les formes en HAVE GOT, les formes initiées par Thomas son majoritaires avec $68 \%$ des occurrences, les formes de reprise ne représentant que $32 \%$ des occurrences en HAVE GOT. Contrairement aux formes en HAVE $+\mathrm{EN}$ où Thomas se situe largement dans la répétition, il jouit d'une plus grand autonomie dans les formes en HAVE GOT. Cette forme étant une forme fixe, Thomas peut l'utiliser à sa guise une fois qu'il a intégré sa morphologie et son sémantisme; l'expression de la possession est par ailleurs très importante dans le développement psycholinguistique de l'enfant (Leroy-Collombel, M. \& Morgenstern, A., 2012) puisqu'il peut se comparer à d'autres individus et se poser lui-même comme possesseur, et donc à ce titre, exister et jouer un rôle dans sa communauté linguistique. Par rapport au total des 130 occurrences, ce sont les formes de reprise en HAVE + EN qui représentent le pourcentage le plus élevé avec $35 \%$. On remarque ainsi que le PP, comme bien d'autres formes dans l'acquisition du langage, est d'abord une forme qui est reproduite, dupliquée (la première forme de PP que nous avons trouvée chez Thomas est d'ailleurs extraite d'une chanson apprise qu'il répète). Cette forme dupliquée est ensuite assimilée par l'enfant au fur et à mesure de ses 
propres productions mais aussi des interactions avec l'adulte ou plus largement avec son environnement linguistique.

L'analyse des résultats quantitatifs nous offre donc la possibilité d'obtenir un schéma global, une sorte de " profil linguistique » de Thomas en relation avec l'étude du PP. On obtient un aperçu de ce qu'il maîtrise et ce qu'il est en voie de maitriser et de la place de l'input dans ses productions.

\subsection{Résultats qualitatifs}

\subsubsection{Développement morpho-syntaxique}

La première forme de PP que l'on trouve chez Thomas se situe à 2 ans 4 mois ("Where have you been?» adressé à son chat Purdie). Cette forme est probablement une expression figée, une structure empruntée au langage entendu sans être encore vraiment analysée par Thomas. Entre l'apparition de cette forme et l'âge de 2 ans 8 mois, la morpho-syntaxe est très instable; l'auxiliaire HAVE ou le participe passé sont parfois erronés et la phonétique est approximative. À partir de 2 ans et 8 mois, la phonétique se stabilise et les formes également. On assiste, à partir de 2 ans et 8 mois, à une surgénéralisation de l'auxiliaire HAVE aux dépens de l'auxiliaire DO qui ne semble pas encore être maîtrisé :

Âge 2;10

MOT: Do you know what I mean?

CHI : No, I haven't.

\subsubsection{Développement sémantique} concrets (I have got twenty pence, 3 ans 1 mois) ou métaphoriques (I haven't got chicken pox, 3 ans 4 mois). L'utilisation de ces deux types de compléments montre que Thomas a saisi une certaine richesse sémantique proposée par l'expression de la possession mais qu'il utilise aussi le langage pour accéder au monde de la représentation, ce qui est un stade important dans son développement psychologique.

classification sémantique de Comrie (voir supra 1.2), deux catégories ressortent nettement chez Thomas : le PP de résultat avec $48 \%$ et le PP d'expérience avec $43 \%$; le PP de passé récent ne représente que $9 \%$ des occurrences et il n'y a aucune occurrence pour le PP de situation persistante. Le PP de passé récent et le PP de situation persistante sont des formes plus complexes que le PP de résultat et le PP d'expérience puisque prototypiquement, le PP de passé récent requiert un adverbe tel que « just » et le PP de situation persistante une forme progressive en -ING combinée à la forme au PP. Il serait utile d'analyser les occurrences de l'input, ou au moins de faire une moyenne, pour voir si l'on retrouve ces pourcentages.

\subsubsection{Fonctions pragmatiques}

Les fonctions pragmatiques du PP trouvées chez Thomas sont au nombre de trois ; nous avons illustré chaque fonction par un exemple de notre corpus, représentatif de la totalité des occurrences :

1) commenter un événement (valeur résultative) : 
Âge $2 ; 8$

MOT: There we are. I've posted another letter through the slot.

$\mathrm{CHI}$ : The letter has gone now. This letter's gone. globalité. Sa mère emploie elle aussi un PP («I've posted») pour montrer que l'événement de poster la lettre est terminé. Thomas s'intéresse quant lui à la lettre en elle-même et déduit qu'elle ne reviendra pas, elle est partie définitivement. Les enfants en acquisition du langage sont très sensibles à l'apparition ou la disparition visuelle d'objets comme c'est le cas ici. Antinucci \& Miller (1976) nous disent qu'un enfant a besoin littéralement de "voir» la complétude d'un événement pour l'encoder au passé. Ici, Thomas l'encode au PP pour marquer la rupture temporelle (la lettre n'est plus là) mais aussi pour marquer le résultat (elle ne reviendra pas). Il est également à noter que dans les premiers emplois des verbes "done» et "gone", la différence est parfois difficilement perceptible chez Thomas, il ne distingue pas encore clairement les deux. Ils sont transcrits selon le contexte et l'audio nous est très précieux pour clarifier les occurrences.

2) chercher une réaction du co-énonciateur (ce qui pourrait revenir à une négociation du thème) :

Âge 2;9

MOT: Come and look all the things [..] on the post office counter.

CHI: I've found something! Your parcel!

Jouer au postier constitue la situation d'énonciation. Puisque c'est un jeu, il faut inventer des scénarios et diversifier les activités. La mère de Thomas attire son attention sur tous les objets présents sur le comptoir, elle lui demande même de venir les regarder (« come and look»). Dans le co-texte gauche, Thomas s'amuse à remplir son camion de colis. En s'exclamant avoir trouvé le colis de sa mère, il opère une négociation du thème puisqu'il veut parler du colis de sa mère, non pas des colis du comptoir ou des colis de son camion même si le colis de sa mère provient probablement du comptoir. Grâce à l'utilisation du $\mathrm{PP}$, il demande à son interlocuteur de changer de conversation et selon la formulation de Benveniste (1966), « établit un lien vivant entre l'événement passé (il a trouvé le colis) et le présent où son évocation trouve place » (la situation d'énonciation qui est maintenant affectée par cet événement passé).

3) partager son expérience:

Âge 2;10

MOT: Who is Henry then?

CHI : I can't see Henry.

MOT: Can't you?

CHI : No.

CHI : I haven't seen him.

Le thème de la conversation est Henry, le personnage d'un jeu. Thomas est en train de jouer à un jeu où il y a différents personnages, Doug, Percy...il accumule les prénoms dans cette scène et s'y perd un peu. Lorsque sa mère lui demande qui est Henry, Thomas commence par se servir de sa perception visuelle, il ne le voit pas. On trouve dans le cotexte gauche un événement «I can't see him » qui garantit la vérité de son énoncé au PP «I haven't seen him » (« commonsense entailment » dans la classification de Nishiyama \& Koening, 2005 ; voir supra 1.3.). C'est parce Thomas n'a pas vu Henry qu'il en déduit qu'il n'en a aucune connaissance, cela n'appartient pas à son expérience, le verbe « see » dans «I haven't seen him » prend alors un sémantisme plus large, il signifie « rencontrer » plus

Corela, HS-13 | 2013 
que "voir». Thomas n'a pas fait sa connaissance, il ne sait pas qui il est et clôt ainsi la conversation puisque le thème de la conversation lui est inconnu, on peut aussi analyser cet exemple comme une négociation du thème: Thomas se sert de son expérience (ici non-expérience) pour clore la conversation et passer à autre chose.

\section{Conclusion} méthodologiques utiles. CLAN permet de mener des analyses quantitatives, d'avoir accès à l'audio et au non-verbal grâce aux annotations sur le corpus. Un corpus longitudinal permet de suivre le développement de la morpho-syntaxe, des fonctions cognitives (très importantes pour le développement de la pragmatique) et de suivre l'entourage de l'enfant (input) et sa mémoire événementielle, la notion d'expérience étant fondamentale dans une étude de l'acquisition du PP. Le codage EXCEL, inspiré par l'équipe CoLaJE (Morgenstern, 2009), permet de combiner l'analyse qualitative des transcriptions, des fichiers audio/vidéo et des analyses quantitatives. Une classification ordonnée et visuelle des données est possible, les analyses quantitatives sont facilitées et des occurrences qui pourraient se prêter à une analyse qualitative plus fine peuvent être rapidement retrouvées. Les premiers résultats que nous avons obtenus nous permettent de mieux comprendre le développement linguistique (qu'il soit sémantique, syntaxique, phonologique ou pragmatique) de l'enfant mais aussi le rapport de ces développements avec l'émergence de nouvelles capacités cognitives et discursives. Le PP n'apparait pas comme un simple marqueur grammatical pour l'enfant ; c'est déjà un « signe » chargé de sens et utilisé dans des contextes bien précis, que nous avons tenté d'identifier.

Pour étoffer et rendre l'étude plus exhaustive et précise, il serait nécessaire de coder les autres formes verbales pour faire ressortir les spécificités du PP et il serait également intéressant de coder les formes de PP trouvées dans l'input pour les comparer à celles de Thomas. Nous pourrions ainsi examiner comment l'enfant s'approprie ce qu'il reçoit et jusqu'à quel point sa production se rapproche de l'input.

\section{BIBLIOGRAPHIE}

Antinucci, F. \& Miller, R. (1976). "How children talk about what happened". Journal of Child Language. 3, 169-189.

Aristote. Métaphysique, traduit par Bernard Sichère, (2 vol. ), Livres A à E. Paris : Pocket, coll. Agora, 2007. Livres Z à N. Paris: Pocket, coll. Agora, 2010.

Bardovi-Harlig, K. (2000). Tense and Aspect in Second Language Acquisition: Form, Meanings, and Use. Oxford: Blackwell.

Benveniste, E. (1966). Problèmes de linguistique générale, volume 1. Collection TEL. Paris: Gallimard.

Corela, HS-13 | 2013 
Bloom, L., Lifter, K. \& Hafitz, J. (1980). "Semantics of verbs and the development of verb inflection in child language". Language, 56, 386-412.

Bronckart, J-P. \& H. Sinclair (1973). "Time, tense and aspect". Cognition, 2, 107-130.

Brown, R. (1973). A First Language: The Early Stages. Cambridge, MA: Harvard University Press.

Collins, A. M. \& Loftus, E. F. (1975). "A spreading-activation theory of semantic processing". Psychological Review, 82, 407-428.

Comrie, B. (1976). Aspect. New York: Cambridge University Press.

Cromer, R.F. (1968). The Development of Temporal Reference during the Acquisition of Language.

Unpublished doctoral dissertation, Harvard University.

Hundt, M. \& Smith, N. (2009). "The present perfect in British and American English: Has there been any change, recently?" ICAME Journal, 33, 45-63.

Lancaster, J.S. \& Barsalou, L.W. (1997). "Multiple organisations of events in memory". Memory, 5, 569-599.

Leech, G.N. (1971). Meaning and the English Verb. London: Longman.

Leroy-Collombel, M. \& Morgenstern, A. (2012). "Rising grammatical awareness in a Frenchspeaking child from 18 months to 36 months: uses and misuses of possession markers". French Language Studies, 22(1), 57-75, Cambridge: Cambridge University Press.

Li, P. \& Shirai, Y. (2000). The Acquisition of Lexical and Grammatical Aspect. Berlin \& New York: Mouton de Gruyter.

Lieven, E., Salomo, D. \& Tomasello, M. (2009). "Two-year-old children's production of multiword utterances: A usage-based analysis". Cognitive Linguistics, 20(3), 481-508.

Macwhinney, B. (2000). The CHILDES Project: Tools for Analyzing Talk. Mahwah, NJ: Erlbaum.

Madden, C. J. \& Zwaan, R. A. (2003). "How does verb aspect constrain event representations?" Memory and Cognition, 31, 663-672.

Maslen, R., Theakston, A., Lieven, E. \& Tomasello M. (2004). "A dense corpus study of past tense and plural overregularization in English". Journal of Speech, Language, and Hearing Research, 47, 1319-1333.

McCawley, J. (1971). "Tense and time reference in English". In C.J. Fillmore \& D.T. Langendoen (eds). Studies in Linguistic Semantics. New York: Holt, Rinehart and Winston, 96-113.

McCoard, R. (1978). The English Perfect : Tense-choice and Pragmatic Inferences. Amsterdam : NorthHolland Publishing Company.

Morgenstern, A. \& Parisse, C. (2007). "Codage et interprétation du langage spontané d'enfants de 1 à 3 ans". Corpus, 6 "Interprétation, contextes, codage", 55-78.

Morgenstern, A. (2009). L'enfant dans la langue. Paris : Presses Universitaire de la Sorbonne Nouvelle.

Nishiyama, A. \& Koening, J-P. (2005). "The discourse functions of the present perfect". In A. Benz and P. Kuehnlein (eds) Constraints in Discourse. John Benjamins : Amsterdam. 201-223.

Nussbaum, N.J. \& Narremore R.C. (1975). "On the acquisition of present perfect 'have' in normal children". Language and Speech, 18, 219-26. 
Parisse, C. \& Morgenstern, A. (2012). "The unfolding of the verbal temporal system in 2 French children's speech between 18 and 36 months". French Language Studies 22(1), 95-114, Cambridge: Cambridge University Press.

Rowland, C. F. \& Fletcher, S. L. (2006). "The effect of sampling on estimates of lexical specificity and error rates". Journal of Child Language, 33, 859-877.

Shirai, Y. \& Andersen, R. W. (1995). "The acquisition of tense-aspect morphology: A prototype account". Language, 71, 743-762.

Slobin, D.I. (1994). "Talking perfectly: Discourse origins of the present perfect". In W. Pagliuca (ed.), Perspectives on Grammaticalization. Amsterdam \& Philadelphia: John Benjamins, 119-133.

Smith, C. (1991). The Parameter of Aspect, Amsterdam : Kluwer Academic Publishers.

Tomasello, M. (2003). Constructing a Language: A Usage-Based Theory of Language Acquisition. Cambridge, MA: Harvard University Press.

Vu, H., Kellas, G., Petersen, E. \& Metcalf, K. (2003). "Situation-evoking stimuli, domain of reference, and the incremental interpretation of lexical ambiguity". Memory \& Cognition, 31, 3021315.

Wagner, L. (1997). Acquiring viewpoint aspect one level at a time. Institute for Research in Cognitive Science Report 97-01, University of Pennsylvania.

Wagner, L. (2001). "Aspectual influences on early tense comprehension". Journal of Child Language, $28,661-681$.

Wagner, L. (2002). "Understanding completion entailments in the absence of agency cues". Journal of Child Language, 29, 109-125.

Weist, R.M. (1991). "Spatial and temporal location in child language". First Language, 11, 253-267.

Weist, R.M. (2002). "The first language acquisition of tense and aspect". In R. Salaberry \& Y. Shirai (eds.), The L2 Acquisition of Tense-Aspect Morphology. Amsterdam: Benjamins, 25-78.

\section{RÉSUMÉS}

Le Present Perfect est une combinaison grammaticale qui regroupe des propriétés temporelles, sémantiques et pragmatiques, ce qui paraît particulièrement complexe dans l'acquisition du langage. Suite aux nombreux travaux sur l'acquisition du groupe verbal, l'étude de l'émergence et du développement du Present Perfect peut nous permettre de mieux comprendre la nature de cette construction et de savoir comment elle apparaît et se complexifie dans l'esprit de l'enfant. Dans cet article, diverses approches théoriques sont présentées et mises en rapport avec l'analyse d'un corpus conversationnel dont le choix est justifié. La méthodologie, la mise en place du protocole et du codage des données sont présentées pour mettre en lumière le travail de regroupement et de classification des données. Les premiers résultats obtenus sont également analysés et discutés dans le but de montrer l'intérêt de mener conjointement des analyses quantitatives et qualitatives multimodales.

The Present Perfect is a grammatical combination that encompasses temporal, semantic and pragmatic properties. This seems to be particularly challenging in language acquisition. Considering the numerous works on the acquisition of the verb phrase, the study of the emergence and the development of the Present Perfect can help us better understand the nature of this construction and it can enable us to seize how it appears and expands in the child's speech. 
In this article, we introduce diverse theoretical approaches and we account for the choice of a conversational corpus that we study in the light of our theoretical background. We examine the methodology and the choice of an appropriate protocol and coding of the data to show how we organise and classify the data. The first results are also analysed and discussed in order to show the importance of having both quantitative and qualitative multimodal analyses.

INDEX

Mots-clés : acquisition du langage, Present Perfect, anglais britannique, corpus dense, méthodologie

Keywords : language acquisition, British English, dense corpus, methodology

\section{AUTEUR}

\section{LAURENT DAVID}

Université Sorbonne Nouvelle, Paris 3 - PRISMES

laurent.david@univ-paris3.fr 